\title{
Developing the surgical technique reporting checklist and standards: a study protocol
}

Kaiping Zhang ${ }^{1,2} \wedge$, Yanfang $\mathrm{Ma}^{3}$, Qianling Shi ${ }^{4}$, Jinlin $\mathrm{Wu}^{5}$, Jianfei Shen ${ }^{6}$, Yulong $\mathrm{He}^{4}$, Xianzhuo Zhang ${ }^{4}$, Panpan Jiao ${ }^{4}$, Grace S. Li ${ }^{1}$, Xueqin Tang ${ }^{1}$, Rene Horsleben Petersen ${ }^{7}$, Calvin S. H. Ng ${ }^{8}$, Alfonso Fiorelli', Nuria M. Novoa ${ }^{10}$, Benedetta Bedetti ${ }^{11}$, Giovanni Battista Levi Sandri ${ }^{12}$, Steven Hochwald ${ }^{13}$, Toni Lerut ${ }^{14}$, Alan D. L. Sihoe ${ }^{15}$, Leandro Cardoso Barchi ${ }^{16}$, Sebastien Gilbert ${ }^{17}$, Ryuichi Waseda ${ }^{18}$, Alper Toker $^{19}$, Diego Gonzalez-Rivas ${ }^{20,21}$, Robert Fruscio ${ }^{22,23}$, Marco Scarci ${ }^{24}$, Fabio Davoli ${ }^{25}$, Guillaume Piessen ${ }^{26}$, Bin Qiu $^{27}$, Stephen D. Wang ${ }^{1}$, Yaolong Chen ${ }^{28,29}$, Shugeng Gao ${ }^{27}$

${ }^{1}$ Editorial Office, AME Publishing Company, Hong Kong, China; ${ }^{2}$ School of Public Health, Imperial College London, London, UK; ${ }^{3}$ EvidenceBased Medicine Center, School of Basic Medical Sciences, Lanzhou University, Lanzhou, China; ${ }^{4}$ The First School of Clinical Medicine, Lanzhou University, Lanzhou, China; ${ }^{5}$ Department of Cardiovascular Surgery, Guangdong Provincial People's Hospital, Guangdong Academy of Medical Sciences, Guangdong, China; ${ }^{6}$ Department of Cardiothoracic Surgery, Taizhou Hospital of Zhejiang Province Affiliated to Wenzhou Medical University, Linhai, China; ${ }^{7}$ University Hospital of Copenhagen, Rigshospitalet, Copenhagen, Denmark; ${ }^{8}$ The Chinese University of Hong Kong, Prince of Wales Hospital, Shatin, New Territories, Hong Kong, China; ${ }^{9}$ Thoracic Surgery Unit, University of Campania "Luigi Vanvitelli”, Naples, Italy; ${ }^{10}$ Thoracic Surgery Service, University Hospital of Salamanca, Biomedical Institute of Salamanca, Salamanca, Spain; ${ }^{11}$ Department of Thoracic Surgery, Helios Clinic Bonn/Rhein Sieg, Bonn, Germany; ${ }^{12}$ Division of General Surgery and Liver Transplantation, S. Camillo Hospital, Rome, Italy; ${ }^{13}$ Department of Surgical Oncology, Roswell Park Cancer Institute, Buffalo, NY, USA; ${ }^{14}$ Department of Thoracic Surgery, University Hospital Leuven, Leuven, Belgium; ${ }^{15}$ Gleneagles Hong Kong Hospital, Hong Kong, China; ${ }^{16}$ Digestive Surgery Division, Department of Gastroenterology, University of Sao Paulo School of Medicine, São Paulo, Brazil; ${ }^{17}$ Division of Thoracic Surgery, Department of Surgery, The Ottawa Hospital, University of Ottawa, Ottawa, Canada; ${ }^{18}$ Department of General Thoracic, Breast and Pediatric Surgery, Fukuoka University, Fukuoka, Japan; ${ }^{19}$ Department of Cardiovascular and Thoracic Surgery, West Virginia University Heart and Vascular Institute, Morgantown, WV, USA; ${ }^{20}$ Department of Thoracic Surgery and Lung Transplant, Coruña University Hospital, Minimally Invasive Thoracic Surgery Unit (UCTMI), Coruña, Spain; ${ }^{21}$ Department of Thoracic Surgery, Shanghai Pulmonary Hospital, Tongji University School of Medicine, Shanghai, China; ${ }^{22}$ Clinic of Obstetrics and Gynecology, San Gerardo Hospital, Monza, Italy; ${ }^{23}$ Department of Medicine and Surgery, University of Milan-Bicocca, Milan, Italy; ${ }^{24}$ Department of Thoracic Surgery, S. Gerardo Hospital, Monza, Italy; ${ }^{25}$ Department of Thoracic Surgery, AUSL Romagna, S. Maria delle Croci Teaching Hospital, Ravenna, Italy; ${ }^{26}$ University of Lille, Department of Digestive and Oncological Surgery, Claude Huriez University Hospital, Lille, France; ${ }^{27}$ Department of Thoracic Surgery, National Cancer Center/National Clinical Research Center for Cancer/Cancer Hospital, Chinese Academy of Medical Sciences and Peking Union Medical College, Beijing, China; ${ }^{28}$ World Health Organization (WHO) Collaborating Centre for Guideline Implementation and Knowledge Translation, Lanzhou, China; ${ }^{29}$ Lanzhou University Institute of Health Data Science, Lanzhou, China Contributions: (I) Conceptualization and design: SD Wang, Y Chen, K Zhang; (II) Administrative support: SD Wang; (III) Provision of study material or patients: None; (IV) Collection and assembly of data: K Zhang, Y Ma, Q Shi; (V) Data analysis and interpretation: K Zhang, Y Ma, Q Shi, X Zhang, P Jiao; (VI) Manuscript writing: All authors; (VII) Final approval of manuscript: All authors.

Correspondence to: Yaolong Chen, MD, MSc., MBBS. Evidence-Based Medicine Center, School of Basic Medical Sciences, Lanzhou University, No.199 Donggang West Road, Lanzhou, China. Email: chenyaolong@lzu.edu.cn; Shugeng Gao, MD. Department of Thoracic Surgery, National Cancer Center/National Clinical Research Center for Cancer/Cancer Hospital, Chinese Academy of Medical Sciences and Peking Union Medical College, No.17 Panjiayuan Nanli, Beijing 100021, China. Email: gaoshugeng@vip.sina.com.

Background: Standardized and transparent reporting of surgical technique is the cornerstone of effective dissemination, implementation and improvement. However, current reporting of surgical techniques is inadequate. The existing guidelines potentially applied to guide surgical technique reporting are with a minimal highlight of the surgical technique, lack requirements explaining what extent and dimensions need to be described in detail, or are unlikely to extrapolate to a wide range of surgical techniques. This study aims

^ ORCID: 0000-0002-7645-2631. 
to formulate a rigorous protocol to develop a surgical technique reporting checklist and standards (SUPER) that defines what a clear, comprehensive and detailed surgical technique report should be contained.

Methods: This protocol is designed following the classic guidance for developing reporting guidelines recommended by the EQUATOR network.

Results: The development team will consist of surgeons ( $80 \%)$, methodologists, and journal editors. The draft checklist sources will include a scoping review of existing reporting guidelines related to surgical technique, surgical technique articles from 15 top journals published in the last year, and brainstorming by the multidisciplinary development team. The final SUPER checklist will be formed after three rounds of Delphi surveys, one round of face-to-face meeting, and a month-long pilot test. The SUPER checklist will be published as open-access and be used in combination with existing reporting guidelines related to surgical techniques (e.g., IDEAL). This protocol will steer the SUPER checklist's development, allowing us to further elaborate surgical technique reporting for all surgical specialties, and enabling a more favorable experience for surgeons, nurses, medical students, residents, editors, and reviewers.

Trial Registration: This trial is registered at the EQUATOR network on December 18th, 2020. Available at: https://www.equator-network.org/library/reporting-guidelines-under-development/reporting-guidelinesunder-development-for-other-study-designs/

Keywords: Surgical technique; reporting checklist; protocol; surgical technique reporting checklist and standards (SUPER)

Submitted May 10, 2021. Accepted for publication Jul 14, 2021.

doi: $10.21037 / g s-21-312$

View this article at: https://dx.doi.org/10.21037/gs-21-312

\section{Introduction}

Although frequently used, a clear definition of "surgical technique" was not found in either the literature or dictionary. Based on the Oxford dictionary definitions of "surgical" and "technique" (1) and the scenarios in which "surgical technique" is used in the literature, we define "surgical technique" as "the specific way and skills of performing a particular medical operation".

Surgical technique is taught and inspired by a master, the so-called "apprenticeship model" introduced by William Halsted a century ago. As an essential component of surgery, a new surgical technique is introduced and disseminated through medical reports, books, live or recorded surgical videos at academic conferences, videos shared on social media and databases, medical labs, etc. However, many books cannot represent the best cutting-edge surgical technique due to the nature of the time-consuming publication. Live or recorded surgical videos at academic conferences are also constrained in time, geography, funding, need for active participants, and high requirements for network speed. Videos that share surgical technique on social media platforms such as LinkedIn (2), YouTube (3) and databases such as CTSNet (4) are not peer-reviewed.
In contrast, surgical technique literature is cutting-edge, widely accessible, and peer-reviewed, and serves as a vital form of surgical technique presentation, dissemination, implementation, evaluation and improvement. Standardized reporting of surgical technique in the literature by reporting guidelines is the cornerstone of effective dissemination and implementation, objective evaluation and continuous improvement. It is gratifying that several reporting guidelines that could be applied to surgical technique have been developed since the publication of CONSORT (5). For instance, CARE and SCARE can be applied in case reports $(6,7)$, PROCESS (8) in case series, STROCSS (9) in cohort studies, CONSORT-NPT (10) in nonpharmacological randomized controlled trials (RCTs), IDEAL (11) in the surgical innovation development process, TIDieR (12) in interventions in a broad sense coving surgical technique, as well as reporting guidelines related to a specific surgical technique (13-19).

However, although these reporting guidelines which could be applied in surgical technique reports cover many types of studies and certain specialties, no reporting guideline that defines what a clear, comprehensive and detailed surgical technique report should be contained 
was found. Specifically, the existing guidelines either address the entire surgical process with minimal highlight on the surgical technique, or simply propose a detailed description requirement without explaining what extent and which dimensions need to be described in detail, i.e., of the benefits and disadvantages of the reported surgical technique. In addition, guideline descriptions of precise surgical technique are challenging to apply in other techniques. As TIDieR (12) and CONSORT-NPT (10) are not specific for surgical techniques since they were developed from a broad perspective, these two guidelines only require sufficient details to allow replication while they do not give specific instructions. As IDEAL (11) focuses on the entire surgical innovation development trajectory (stage 1 , stage $2 \mathrm{a}$, stage $2 \mathrm{~b}$, stage 3 , and stage 4 ), it is also reasonable that only limited items (item $5 \mathrm{a}$ and item $5 \mathrm{~b}$ ) are related to the surgical technique. Item $7 \mathrm{~d}$ and item $7 \mathrm{e}$ in STROCSS (9) require more detailed surgical technique reporting than TIDieR, CONSORT-NPT, and IDEAL, such as quality control, reduction in operator variation, reporting who performed the surgery, and their learning curve status. Nevertheless, the reporting requirements are far from completeness. Item $4 \mathrm{~g}$, item $4 \mathrm{~h}$, item $4 \mathrm{i}$ in PROCESS (8) and item 9b, item 9c, item 9d in SCARE (6) involves much more detailed requirements for surgical technique, including anesthetic, patient position, use of a tourniquet, and other relevant equipment, the preparation used, sutures, devices, surgical stages, quality control and maintenance of consistency etc. However, PROCESS and SCARE are positioned only for surgical case series and case reports, respectively. Many reporting guidelines covering a specific surgical technique have very elaborated requirements $(13,15,20-22)$, but they lack a consistent framework and are unlikely to extrapolate to other surgical techniques.

Accordingly, due to the lack of surgical technique reporting guidelines, most journals in the surgical field do not have clear instructions of which guideline should be followed in order to describe surgical technique. According to a systematic review published in 2014 (23) which evaluated 193 surgical journals, $62 \%$ of the journals did not mention which reporting guideline were used and $86 \%$ of the journals did not require authors to use any guideline. Furthermore, the classification of surgical technical articles and the specific reporting requirements differed from journal to journal. Only very few journals, such as the Annals of Cardiothoracic Surgery (24) have, to some extent, detailed reporting requirements regarding surgical technique. Specifically, this journal classifies surgical technique in the Art of Operative Techniques category and requires between 9 to 12 medical drawings/photographs with detailed legends together with a description of the operative procedure in a step-by-step manner, and provides separate instructions on standardized structure (25) with a large number of examples (26). Whereas, most other journals have very vague reporting requirements. Two other top journals in cardiothoracic surgery, the Fournal of Thoracic and Cardiovascular Surgery (27) and the European Fournal of Cardio-Thoracic Surgery (28), both concern and classify surgical technique as an independent category article. However, the two journals' reporting requirements are minimal, with only brief requirements regarding the need for surgical images and videos, limitations on the number of figures and videos, and legends. The Annals of Thoracic Surgery places surgical technique in the "How to Do It" and "New technology" category, which similarly has not concrete enough reporting requirements, though authors can illustrate new surgical techniques more extensively in "New technology" (29). Similar issues exist in the top comprehensive surgical journals, such as Annals of Surgery (30) and $7 A M A$ Surgery (31).

Furthermore, the lack of comprehensive reporting guidelines and the corresponding inconsistent journal requirements has resulted in poor surgical technique reporting. Surgical images and videos are either present or absent within the article and surgical videos lose impact due to absence of captions or audios (32). A systematic review (33) which included 158 RCTs in the field of surgical intervention showed that the reporting quality was low, as only $40.5 \%$ of the articles describing how to carry out the intervention. Still, only $32.2 \%$ reported the number of people performing the surgical intervention. In addition, a recent systematic review (34) which evaluated 130 RCTs published in the fifteen top journals in the field of plastic and reconstructive surgery found that the overall reporting score was only 6.4 out of 12 , and four items of TIDieR were reported less than $10 \%$ of the time. The authors also pointed out that due to the complexity of surgical technique from incision to closure, more elaborate reporting guidelines in this area are desperately needed.

Therefore, we aim to develop a surgical technique reporting checklist and standards (SUPER) which could be applied to all surgical specialties. The SUPER checklist is focused on the intraoperative guidelines for the surgical technique rather than focusing on perioperative care, and focused on the treatment of abnormalities in people rather than focusing on diagnosis. It is neither limited to certain 


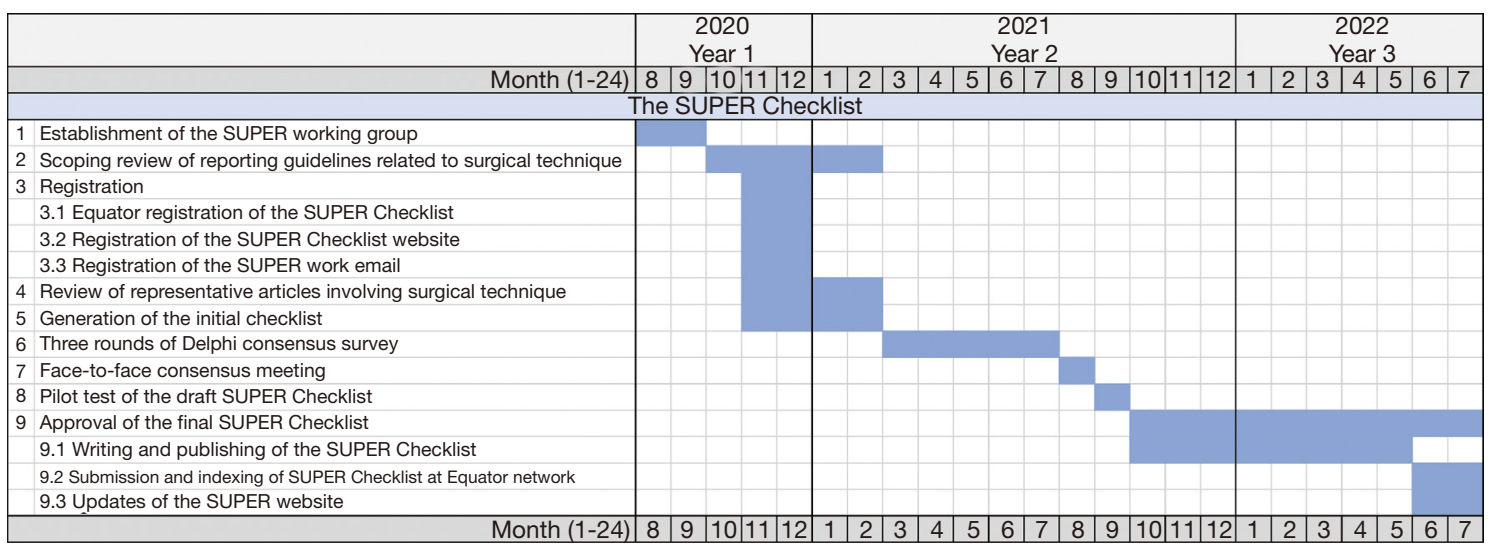

Figure 1 Key steps and timeline of the SUPER checklist development plan. SUPER, surgical technique reporting checklist and standards.

types of articles, such as RCTs, observational studies, case series and case report, nor limited to a particular stage of surgical technique development, such as the invention stage, optimization stage or evaluation stage. The SUPER checklist will allow more complete, logical, transparent and elaborate surgical technique reporting, which will be very helpful to surgeons, residents, medical students, researchers and journal editors. The SUPER checklist will be used to combine existing reporting guidelines, IDEAL or SCARE, CARE, PROCESS, STROCSS, CONSORT-NPT and TIDieR. This protocol features the methodology and steps that will be taken to develop the SUPER checklist.

\section{Methods and timeline}

The SUPER checklist will be developed based on the recommended method by the Equator network (35). Some of the key steps and timeline are summarized in Figure 1. Each will be addressed accordingly.

\section{Initial steps}

\section{Establishment of the SUPER working group}

The SUPER working group will include surgeons, methodologists and journal editors. The majority will be composed of surgeons ( $\sim 80 \%)$. The composition of the working group will take into account appropriate representation from countries/regions, disciplines and specialties. Specifically, for surgeons, we will include junior surgeons who are in the early learning curving of surgery and senior experienced surgeons, and both male and female surgeons. The nationalities of participating surgeons are expected to be distributed over 10 high, middle and low-income countries; for journal editors, we will include academic editors who have professional academic backgrounds and management editors with rich experience in journal management; For methodologists, we will include professional methodologists with evidence-based medicine backgrounds and methodologists with both clinical and evidence-based medicine backgrounds. All these considerations are aimed to establish a broad and representative group and gain representative opinions.

Each SUPER working group member must sign a conflict of interest (COI) declaration form and a confidentiality agreement. The group will establish a COI management team consisting of five experts, including a principal investigator, a methodologist, two senior surgeons and an ethicist. Only those endorsed by the COI management team can join the development of the SUPER checklist. The endorsement will identify COI at the academic and non-academic aspects, as well as the individual and institutional aspects. The management of COI are as follows: (I) members with severe COI will be prohibited from participating in the SUPER checklist's entire development; (II) members with a partial COI will be evaluated on the degree and to the specific area of the COI and will be recused from the appropriate development session; (III) members with a minimal COI that do not affect the objectivity of the development may participate in the development. The COI forms for each member who have participated in the SUPER development will be publicly available.

The SUPER working group will include two subgroups, 
Table 1 Search strategy of reporting guidelines related to surgical technique (MEDLINE)

\begin{tabular}{|c|c|}
\hline Number & Searches \\
\hline$\# 1$ & "reporting guideline" [Title/Abstract] \\
\hline$\# 2$ & "reporting requirement ${ }^{\star ”}[$ Title/Abstract] \\
\hline \#3 & "research reporting" [Title/Abstract] \\
\hline \#4 & “minimum information" [Title/Abstract] \\
\hline \#5 & "guideline*" [Title] AND “reporting" [Title] \\
\hline \#6 & $\begin{array}{l}\text { "guidance" [Title/Abstract] AND "reporting" } \\
\text { [Title/Abstract] }\end{array}$ \\
\hline \#7 & $\begin{array}{l}\text { "transparen"” [Title/Abstract] AND "reporting" } \\
\text { [Title/Abstract] }\end{array}$ \\
\hline \#8 & "guideline*" [Title] AND "publication*" [Title] \\
\hline$\# 9$ & "standard" [Title] AND “reporting" [Title] \\
\hline$\# 10$ & "practice" [Title] AND "reporting" [Title] \\
\hline$\# 11$ & "design" [Title] AND "reporting" [Title] \\
\hline$\# 12$ & "conduct" [Title] AND "reporting" [Title] \\
\hline$\# 13$ & "criteri"” [Title] AND "reporting" [Title] \\
\hline$\# 14$ & "recommendation*" [Title] AND "reporting" [Title] \\
\hline \#15 & "analys" [Title] AND "reporting" [Title] \\
\hline \#16 & "method*" [Title] AND "reporting" [Title] \\
\hline$\# 17$ & "experiment" [Title] AND "reporting" [Title] \\
\hline$\# 18$ & "responsible" [Title] AND "report"” [Title] \\
\hline \#19 & "clarity" [Title] AND "report"” [Title] \\
\hline \#20 & "presentation" [Title] AND "publication" [Title] \\
\hline \#21 & "presentation" [Title] AND "standard"” [Title] \\
\hline \#22 & "presentation" [Title] AND "guideline*” [Title] \\
\hline \#23 & OR/\#1-\#22 \\
\hline \#24 & "Surgical Procedures, Operative"[Mesh] \\
\hline \#25 & "surgery" [Title/Abstract] \\
\hline \#26 & "surgical" [Title/Abstract] \\
\hline \#27 & "operat"” [Title/Abstract] \\
\hline \#28 & "technique" [Title/Abstract] \\
\hline \#29 & "procedure*" [Title/Abstract] \\
\hline$\# 30$ & OR/\#24-\#29 \\
\hline \#31 & \#23 AND \#30 \\
\hline \#32 & Filters: Humans \\
\hline
\end{tabular}

the secretary group and the Delphi panelists. The secretary group will focus on drafting the initial SUPER checklist and report progress weekly. All work and meeting documentation will be fully archived and backed up. The Delphi panelists will mainly focus on three rounds of Delphi consensus survey.

\section{Scoping review of reporting guidelines related to surgical technique}

A systematically search for existing English reporting guidelines related to surgical technique from Equator library, MEDLINE (via PubMed), Google Scholar and Networked Digital Library of Theses and Dissertations (NDLTD) will be performed. The search strategy for MEDLINE (via PubMed) is shown in Table 1.

A scoping review of the retrieved reporting guidelines will then be performed to gain deep, qualitative and quantitative understanding of the current gap for reporting items in the surgical technique field. From this, a more targeted reporting checklist can be proposed.

\section{Registration}

The SUPER checklist project will be registered at the Equator network as soon as the first SUPER checklist protocol draft is completed. To inform more peers and receive feedback and suggestions, registration and construction of the SUPER checklist website will be conducted simultaneously. Progress of the SUPER project will be updated at the SUPER checklist website. A SUPER working group mailbox used for all communication related to the SUPER project will also be registered simultaneously. The staff will be assigned to check and reply to emails regularly.

\section{Review of representative articles involving surgical technique}

It is planned to search (MEDLINE via PubMed), download and review the articles involving surgical technique from the fifteen top journals in the field of surgery and biomedicine. Considering the advantage of $\mathrm{H} 5$ index and quartile rank in selecting representative journals, Google Scholar and Journal Citation Report are chosen as two journal sources. Five general clinical journals are chosen based on the H5 index rank in Google Scholar in the area 'Health \& Medical Sciences'; five general surgical journals are chosen based on 
Table 2 Search strategy of articles related to surgical technique published on the fifteen chosen top journals (MEDLINE)

\begin{tabular}{|c|c|}
\hline Number & Searches \\
\hline$\# 1$ & "Surgical Procedures, Operative"[Mesh] \\
\hline \#2 & "surgery" [Title/Abstract] \\
\hline \#3 & "surgical" [Title/Abstract] \\
\hline \#4 & "operat" [Title/Abstract] \\
\hline \#5 & "technique" ${ }^{\star}$ Title/Abstract] \\
\hline \#6 & "procedure*" [Title/Abstract] \\
\hline \#7 & OR/\#1-\#6 \\
\hline \#8 & "The New England journal of medicine"[Journal] \\
\hline \#9 & "JAMA" [Journal] \\
\hline$\# 10$ & "Lancet (London, England)"[Journal] \\
\hline$\# 11$ & $\begin{array}{l}\text { "Journal of clinical oncology: official journal of the } \\
\text { American Society of Clinical Oncology"[Journal] }\end{array}$ \\
\hline$\# 12$ & "The Lancet. Oncology"[Journal] \\
\hline$\# 13$ & "Annals of surgery"[Journal] \\
\hline$\# 14$ & "JAMA surgery"[Journal] \\
\hline \#15 & "The British journal of surgery"[Journal] \\
\hline \#16 & $\begin{array}{l}\text { "Journal of neurology, neurosurgery, and } \\
\text { psychiatry"[Journal] }\end{array}$ \\
\hline$\# 17$ & "Hepatobiliary surgery and nutrition"[Journal] \\
\hline \#18 & $\begin{array}{l}\text { "Digestive endoscopy: official journal of the Japan } \\
\text { Gastroenterological Endoscopy Society"[Journal] }\end{array}$ \\
\hline \#19 & $\begin{array}{l}\text { "The Journal of bone and joint surgery. American } \\
\text { volume"[Journal] }\end{array}$ \\
\hline \#20 & $\begin{array}{l}\text { "The Journal of thoracic and cardiovascular } \\
\text { surgery"[Journal] }\end{array}$ \\
\hline$\# 21$ & "Journal of Vascular Surgery"[Journal] \\
\hline \#22 & "Annals of Surgical Oncology"[Journal] \\
\hline \#23 & OR/\#8-\#22 \\
\hline \#24 & $\begin{array}{l}\text { (“2019/12/1”[Date - Publication]: "2020/11/30" } \\
\text { [Date - Publication]) }\end{array}$ \\
\hline \#25 & \#7 AND \#23 AND \#24 \\
\hline \#26 & Filters: Humans \\
\hline
\end{tabular}

the H5 index rank in Google Scholar in the 'Surgery' area; and five specialty surgical journals are chosen based on the quartile rank in the Journal Citation Report in the 'Surgery' area. In this way, high representation of articles is assured by including articles from top general clinical, top general surgery and top specialty surgical journals. The timeframe will be between December 1st, 2019 and November 30th, 2020. The search strategy is presented in Table 2.

\section{Generation of the initial checklist}

Based on the scoping review of existing reporting guidelines and review of representative articles from the fifteen journals, the working group will debrief in order to reach consensus on the data to be extracted. Two teams will separately extract item entries from matched guidelines and articles. Both teams must include at least one surgeon, one journal editor, and one methodologist. Before the formal extraction, training will be performed to ensure a consistent understanding of each item entry in the extraction table and consistent extraction results from the same articles. The SUPER working group will also debate to identify additional item entries. Finally, the two teams will have a face-to-face discussion to summarize all included items and generate the initial SUPER checklist.

\section{Three rounds of Delphi consensus survey}

After generating the initial SUPER items, the working group will design the Delphi consensus survey questionnaire using a previously reported method (36). Experts will be invited to score each item on a scale from 1 to 5 , corresponding with the lowest to the highest level of importance. Once receiving the experts' scores, the working group will decide on the inclusion and exclusion of each item, with scores of 1 and 2 indicating low importance for inclusion and high recommendation for exclusion, score 3 indicating medium importance with need for discussion to reach consensus, and scores 4 and 5 indicating high importance for inclusion. Consensus on any item is conditional to at least $66 \%$ of the Delphi survey responses having agreed on the rating in the above three categories (scores of 1 and 2, score 3, scores 4 and 5). During round 1 of the Delphi consensus survey, all initial items will be sent for scoring. Experts will be allowed to add new items and comments to each item during round 1 . Round 2 of the Delphi consensus survey will include items that have not reached consensus and new items suggested by respondent(s) during the round 1 . Then, round 3 will involve items that have not reached consensus in round 1 or round 2. Figure 2 summarizes the detailed process of each item based on the score and consensus scenarios during the three rounds of Delphi survey.

During the Delphi consensus survey, (I) one person will generate computerized random serial numbers for experts who agree to join; (II) another person will be responsible for emailing the questionnaire, sending and collecting the COI declaration form and confidentiality agreement, recording when the questionnaire is sent and received, and recording 


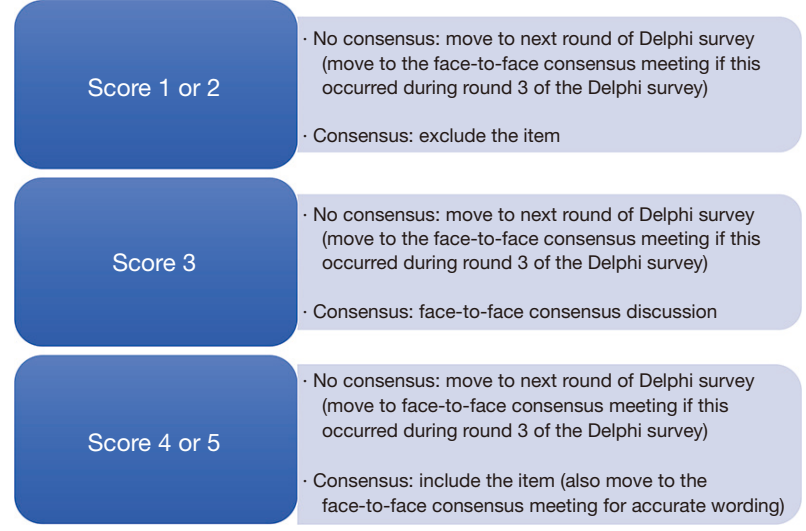

Figure 2 Workflow for each item during the three rounds of Delphi survey.

the response rate; (III) and separate data entry and analysis will be performed by two people. One of the two will be a methodologist, and both will be only aware of the serial numbers and the questionnaire results table.

Finally, the initial checklist that has been agreed upon by the Delphi panelists will be generated.

\section{The face-to-face consensus meeting}

Before the face-to-face consensus meeting, sufficient premeeting preparation will be critical. The participants will include surgeons, anesthetists, nurses, journal editors and methodologists. Surgeons will present more than $25 \%$ of all participants. The checklist after the three rounds of the Delphi survey and existing evidence will be compiled, distributed and discussed at the meeting. Importantly, the whole meeting will be prepared to be recorded in forms of video, audio, and transcript in advance.

Then, a face-to-face offline consensus meeting will be held. The meeting will run for two days, with the firstday sharing existing evidence and the work done to ensure that each attendee has a deep, comprehensive, and clear understanding of the project and items. On the second day, items that need consensus discussion (Figure 2) will be discussed one by one. Consensus on any item is also conditional to at least $66 \%$ agreement of the participants. Finally, a final consensus checklist will be generated.

\section{Pilot test of the SUPER checklist draft}

Based on the consensus checklist generated in the face-toface meeting, the working group will write down a formal reporting checklist and send it to the entire group, including the Delphi panelists, for revision and confirmation.

The checklist will then be sent to the journal editors and surgeons for a pilot test for one month to get feedback and identify any practical challenges, and review clarity of wording and presentation. The working group will actively collect feedback from authors, editors, and surgeons on the pilot test through surveys and questionnaires. Then further refinement of the items will be performed and send for final approval by all the SUPER working group members.

\section{Approval of the final SUPER checklist}

\section{Writing and publishing of the SUPER checklist}

A full SUPER manuscript will be written. The manuscript will provide and list SUPER electronic mailbox to continuously collect feedback and criticism, as well as to indicate a follow-up evaluation and update plan. The explanation and elaboration manuscript will be drafted in parallel for better implementation of the SUPER checklist. Once reviewed by each group member, the manuscripts will be submitted for publication. The manuscripts will be published in an openaccess journal to ensure free access for all readers.

\section{Submission and indexing of SUPER checklist at Equator network}

The SUPER checklist and full manuscript will be submitted to the Equator network once it is published. The working group will make efforts to achieve indexing of the Equator network.

\section{Updates of the SUPER website}

All publications and relevant documents will be uploaded to the SUPER checklist website.

\section{Post-publication activities}

The post-publication activities will occur in four main areas: (I) regularly inquiring about, recording and responding to, feedback and criticism by email and collecting feedback and criticism through other channels, such as literatures and messages, as well as surgeons and journal editors; (II) to vigorously promote dissemination and compliance, including online and offline training sessions for editors and surgical communities, encourage the endorsement from journals, using social media to increase awareness, and through active promotion at surgical conferences; (III) assessing the effect and impact of the SUPER checklist, such as by conducting a 
systematic review; (IV) performing multilingual translations, developing extensions, and updates of the checklist.

\section{Strengths and limitations of the protocol}

The limitations of this protocol include: (I) patient views are not included throughout the development; (II) in order to maximize the applicability of our checklist to all surgical specialties, it may not be possible to provide specific coverage for some very specific or relatively new specialties. However, extensions or expansions of the general checklist are planned at a later stage. Also, updates of subspecialties will be based on the usage and feedback as needed. The strengths are: (I) it is methodologically rigorous and strictly follows the classical method; (II) sufficient diversity in professional background, discipline, country/region, and context is fully considered.

\section{Ethics and dissemination}

As a protocol which design details to develop a checklist, there is no requirement for ethical approval. The protocol and subsequent SUPER checklist will be published as open access. Meanwhile, academic conferences, mainstream media and other channels will be used for broader dissemination.

\section{Acknowledgments}

Funding: This project is supported by the AME Reporting Guidelines Research Fund (No. 2020-1016-885) and Lanzhou University Institute of Health Data Science Fund.

\section{Footnote}

Peer Review File: Available at https://dx.doi.org/10.21037/ gs-21-312

Conflicts of Interest: All authors have completed the ICMJE uniform disclosure form (available at https://dx.doi. org/10.21037/gs-21-312). ADLS receives Speaker's honoraria from Medtronic and is the consultancy of Medela. AF declares a pending patent (PCT/IT2018/00000S) related to the project. FD declares a licensed patent (Italian patent application "TO2013A000038") related to the project. RHP receives speaker fee from Medtronic and is the advisory board member for AstraZeneca. KZ, GSL, $\mathrm{XT}$ and SDW are staff of AME publishing company (the publisher of Gland Surgery). The other authors have no conflicts of interest to declare.
Ethical Statement: The authors are accountable for all aspects of the work in ensuring that questions related to the accuracy or integrity of any part of the work are appropriately investigated and resolved.

Open Access Statement: This is an Open Access article distributed in accordance with the Creative Commons Attribution-NonCommercial-NoDerivs 4.0 International License (CC BY-NC-ND 4.0), which permits the noncommercial replication and distribution of the article with the strict proviso that no changes or edits are made and the original work is properly cited (including links to both the formal publication through the relevant DOI and the license). See: https://creativecommons.org/licenses/by-nc-nd/4.0/.

\section{References}

1. Available online: https://www.oxfordlearnersdictionaries.com/

2. Available online: https://www.linkedin.com/

3. Available online: https://www.youtube.com/

4. Available online: https://www.ctsnet.org/videos

5. Begg C, Cho M, Eastwood S, et al. Improving the quality of reporting of randomized controlled trials. The CONSORT statement. JAMA 1996;276:637-9.

6. Agha RA, Borrelli MR, Farwana R, et al. The SCARE 2018 statement: Updating consensus Surgical CAse REport (SCARE) guidelines. Int J Surg 2018;60:132-6.

7. Gagnier JJ, Kienle G, Altman DG, et al. The CARE guidelines: consensus-based clinical case reporting guideline development. BMJ Case Rep 2013;2013:bcr2013201554.

8. Agha RA, Borrelli MR, Farwana R, et al. The PROCESS 2018 statement: Updating Consensus Preferred Reporting Of CasE Series in Surgery (PROCESS) guidelines. Int J Surg 2018;60:279-82.

9. Agha R, Abdall-Razak A, Crossley E, et al. STROCSS 2019 Guideline: Strengthening the reporting of cohort studies in surgery. Int J Surg 2019;72:156-65.

10. Boutron I, Altman DG, Moher D, et al. CONSORT Statement for Randomized Trials of Nonpharmacologic Treatments: A 2017 Update and a CONSORT Extension for Nonpharmacologic Trial Abstracts. Ann Intern Med 2017;167:40-7.

11. Bilbro NA, Hirst A, Paez A, et al. The IDEAL Reporting Guidelines: A Delphi Consensus Statement Stage Specific Recommendations for Reporting the Evaluation of Surgical Innovation. Ann Surg 2021;273:82-5.

12. Hoffmann TC, Glasziou PP, Boutron I, et al. Better 
reporting of interventions: template for intervention description and replication (TIDieR) checklist and guide. BMJ 2014;348:g1687.

13. Rylski B, Pacini D, Beyersdorf F, et al. Standards of reporting in open and endovascular aortic surgery (STORAGE guidelines). Eur J Cardiothorac Surg 2019;56:10-20.

14. Siriwardena AK, Windsor J, Zyromski N, et al. Standards for reporting on surgery for chronic pancreatitis: a report from the International Study Group for Pancreatic Surgery (ISGPS). Surgery 2020;168:101-5.

15. Timaran CH, McKinsey JF, Schneider PA, et al. Reporting standards for carotid interventions from the Society for Vascular Surgery. J Vasc Surg 2011;53:1679-95.

16. Ahn SS, Rutherford RB, Johnston KW, et al. Reporting standards for infrarenal endovascular abdominal aortic aneurysm repair. Ad Hoc Committee for Standardized Reporting Practices in Vascular Surgery of The Society for Vascular Surgery/International Society for Cardiovascular Surgery. J Vasc Surg 1997;25:405-10.

17. Minervini A, Carini M, Uzzo RG, et al. Standardized reporting of resection technique during nephron-sparing surgery: the surface-intermediate-base margin score. Eur Urol 2014;66:803-5.

18. Patel N, Sacks D, Patel RI, et al. SCVIR reporting standards for the treatment of acute limb ischemia with use of transluminal removal of arterial thrombus. J Vasc Interv Radiol 2001;12:559-70.

19. Reinstein DZ, Archer TJ, Randleman JB. JRS standard for reporting astigmatism outcomes of refractive surgery. $\mathrm{J}$ Refract Surg 2014;30:654-9.

20. Fillinger MF, Greenberg RK, McKinsey JF et al. Society for Vascular Surgery Ad Hoc Committee on TEVAR Reporting Standards. Reporting standards for thoracic endovascular aortic repair (TEVAR). J Vasc Surg 2010;52:1022-33.e15.

21. Morozova TP. Comparison of the blood levels of insulin and growth hormone in healthy adolescents, adolescents with excessive weight and with hereditary loading with regard to diabetes mellitus. Probl Endokrinol (Mosk) 1977;23:3-7.

22. Vanhie A, Meuleman C, Tomassetti C, et al. Consensus on Recording Deep Endometriosis Surgery: the CORDES statement. Hum Reprod 2016;31:1219-23.

23. Agha RA, Barai I, Rajmohan S, et al. Support for reporting guidelines in surgical journals needs improvement: A systematic review. Int J Surg 2017;45:14-7.

24. Available online: http://www.annalscts.com/about/ instructionsForAuthors

25. Available online: http://cdn.amegroups.cn/journals/acs/ public/system/acs/ACS-Art-of-Operative-Techniques.pdf

26. Available online: http://www.annalscts.com/section/view/art

27. Available online: https://www.jtcvs.org/content/authorinfo

28. Available online: https://academic.oup.com/ejcts/pages/ Manuscript_Instructions

29. Available online: https://www.annalsthoracicsurgery.org/ content/authorinfo

30. Available online: http://edmgr.ovid.com/annsurg/accounts/ ifauth.htm

31. Available online: https://jamanetwork.com/journals/ jamasurgery/pages/instructions-for-authors

32. De la Huerta I, Yonekawa Y, Thomas BJ, et al. A Surgical Technique for the Management of Tractional Retinal Detachment in Aggressive Posterior Retinopathy of Prematurity Treated With Intravitreal Bevacizumab. Retina 2019;39 Suppl 1:S156-9.

33. Jacquier I, Boutron I, Moher D, et al. The reporting of randomized clinical trials using a surgical intervention is in need of immediate improvement: a systematic review. Ann Surg 2006;244:677-83.

34. Evans S, Rauh S, Jellison S, et al. Evaluation of the Completeness of Interventions Reported in Published Randomized Controlled Trials in Plastic Surgery: A Systematic Review. Aesthet Surg J 2021;41:707-19.

35. Moher D, Schulz KF, Simera I, et al. Guidance for developers of health research reporting guidelines. PLoS Med 2010;7:e1000217.

36. McInnes MDF, Moher D, Thombs BD, et al. Preferred Reporting Items for a Systematic Review and Metaanalysis of Diagnostic Test Accuracy Studies: The PRISMA-DTA Statement. JAMA 2018;319:388-96.

Cite this article as: Zhang K, Ma Y, Shi Q, Wu J, Shen J, He Y, Zhang X, Jiao P, Li GS, Tang X, Petersen RH, Ng CSH, Fiorelli A, Novoa NM, Bedetti B, Levi Sandri GB, Hochwald S, Lerut T, Sihoe ADL, Barchi LC, Gilbert S, Waseda R, Toker A, Gonzalez-Rivas D, Fruscio R, Scarci M, Davoli F, Piessen G, Qiu B, Wang SD, Chen Y, Gao S. Developing the surgical technique reporting checklist and standards: a study protocol. Gland Surg 2021;10(8):2591-2599. doi: 10.21037/gs-21-312 